E D I T O R I A L S

\title{
Immunotherapy for Unresectable Stage III Non-Small-Cell Lung Cancer
}

\author{
Naiyer A. Rizvi, M.D., and Solange Peters, M.D., Ph.D.
}

In this issue of the Journal, Antonia and colleagues report the results of the phase 3 PACIFIC study, which evaluated the role of immune checkpoint blockade in locally advanced, unresectable, stage III non-small-cell lung cancer (NSCLC). ${ }^{1}$ Eligible patients had disease that had not yet progressed after they had received at least two cycles of platinum-based chemotherapy concurrent with radiotherapy (chemoradiotherapy) at a dose of 54 to $66 \mathrm{~Gy}$. A total of 713 patients were randomly assigned, in a 2:1 ratio, to receive an anti-programmed death ligand 1 (anti-PD-L1) antibody, durvalumab, at a dose of $10 \mathrm{mg}$ per kilogram of body weight or placebo every 2 weeks for up to 12 months.

Worldwide, approximately 500,000 patients receive a diagnosis of unresectable stage III NSCLC every year; this number constitutes approximately one third of all patients with NSCLC. Stage III NSCLC includes either locally advanced primary tumors with infiltration of vital mediastinal organs or involvement of locoregional mediastinal lymph nodes. Over the past decade, chemoradiotherapy has remained the standard of care for stage III NSCLC. Currently, only $15 \%$ of patients are alive 5 years after chemoradiotherapy, and this percentage remains largely unchanged despite multiple phase 3 , randomized trials integrating surgery; increased doses of radiation or consolidation of radiotherapy with chemotherapy; biologic agents; or vaccine treatment. ${ }^{2,3}$

In the study reported by Antonia et al., a preplanned interim analysis showed that the coprimary end point of median progression-free survival was 16.8 months in the durvalumab group versus 5.6 months in the placebo group, corresponding to a hazard ratio for disease progression or death of 0.52 (95\% confidence interval, 0.42 to 0.65 ). The response rate was higher in the durvalumab group than in the placebo group. The robustness and consistency of the results were supported by multiple prespecified sensitivity analyses. Benefit was observed independently of NSCLC disease stage (IIIA or IIIB), histologic type, or geographic distribution.

In a comparable patient population in the RTOG 0617 trial, progression-free survival among patients in the control chemoradiotherapy group was 11.8 months, which was longer than that observed in the placebo group of the PACIFIC study (5.6 months). ${ }^{3}$ However, progression-free survival in the RTOG 0617 trial was calculated from the initiation of chemoradiotherapy. In the Stimulating Targeted Antigenic Response to NSCLC (START) trial, in which randomization occurred after chemoradiotherapy, the progression-free survival among patients in the control group was 8.4 months. ${ }^{4}$ In the study by Antonia et al., randomization occurred after patients had completed chemoradiotherapy and could take place up to 42 days after the end of chemoradiotherapy, allowing for resolution of toxic effects associated with chemoradiotherapy. Factoring in these variables, results in the control group of the PACIFIC study were not inconsistent with data from previous phase 3 trials.

Overall survival continues to be the standard for outcomes in the treatment of locally advanced NSCLC. Nevertheless, in the PACIFIC study, progression-free survival was almost 17 months in 
the durvalumab group, which was almost 1 year longer than progression-free survival in the placebo group; this duration is unprecedented in stage III NSCLC. Additional relevant surrogate end points included the development of new distant lesions that were more frequently observed in the placebo group than in the durvalumab group. Most notably, brain metastasis developed in twice as many patients in the placebo group as in the durvalumab group (11.0\% vs. $5.5 \%$ ). Furthermore, progression-free survival has been found to correlate with overall survival in previous chemoradiotherapy studies. ${ }^{5}$ The overall survival benefit frequently exceeds the progressionfree survival benefit in other studies of immune checkpoint inhibitors that involve patients with advanced-stage NSCLC. ${ }^{6}$ Although the overall survival data in this study have yet to mature, the clinically meaningful difference in progressionfree survival merits consideration of durvalumab as a new standard of care in this patient population.

Given the potential for radiotherapy to increase the likelihood of anti-PD-L1-mediated pneumonitis, uncertainty existed as to the risk of the use of durvalumab. Treatment-related adverse events occurred in $67.8 \%$ of patients in the durvalumab group, as compared with $53.4 \%$ in the placebo group. The rate of immune-mediated adverse events was $24.2 \%$ with durvalumab and $8.1 \%$ with placebo. Treatment was discontinued because of pneumonitis in $6.3 \%$ of patients who received durvalumab and $4.3 \%$ who received placebo. Overall, a slight increase in toxic effects was noted in the durvalumab group, but the rates of severe immune-related adverse events and of pneumonitis, in particular, were not meaningfully different.

Can these results with durvalumab be extrapolated to patients with resectable stage III disease? In the phase 3 Intergroup 0139 trial, the addition of surgery to chemoradiotherapy was not associated with a survival advantage. ${ }^{7}$ Nevertheless, on the basis of retrospective subgroup analyses, standard treatment for "selected" patients with stage IIIA N2 disease (patients with a single nodal station who are candidates for lobectomy and have clearing of mediastinal nodes with induction therapy) involves considering resection after neoadjuvant chemotherapy, although this varies according to geographic location. The data from the PACIFIC study may end the debate about the role of surgery in patients with stage III NSCLC, with durvalumab after chemoradiotherapy redefining best practice for all patients with stage III NSCLC. Of course, this notion will need to be tested prospectively.

Benefit in the PACIFIC study was observed in patients with nonsquamous and squamous tumor histologic types as well as in patients with stages IIIA and IIIB NSCLC. Benefit was also observed irrespective of PD-L1 status. Half the patients enrolled in this study had squamouscell lung cancer, in which the correlation between the PD-L1 biomarker and benefit in advancedstage NSCLC is weak. ${ }^{8}$ Currently, the data support treatment with consolidation in unselected patients. Chemotherapy and radiation therapy can promote immunogenic cell death of tumor cells by activating dendritic cells and enhancing antigen presentation, resulting in induction of adaptive immune responses. These data support the concept of sequencing chemoradiotherapy before blockade of the programmed death 1 pathway. Refinement of this combined strategy regarding the timing and duration of checkpoint-inhibitor intervention will require further evaluation.

Immune checkpoint blockade is currently being evaluated in multiple ongoing clinical trials of neoadjuvant immune checkpoint-blockade approaches in patients with early-stage NSCLC. Blood-based next-generation sequencing of tumor DNA may allow investigators to prospectively identify patients with resectable stage I to III NSCLC who are at the greatest risk for relapse. In a recent study, after resection of NSCLC, at least two detectable single-nucleotide variants were identified preoperatively in 13 of 14 patients with relapse. ${ }^{9}$ The data from the PACIFIC study provide support for the integration of immune checkpoint blockade for unresectable stage III NSCLC and will undoubtedly shape the design of future trials in stage I to III NSCLC.

Disclosure forms provided by the authors are available with the full text of this editorial at NEJM.org.

From Columbia University Medical Center, New York (N.A.R.); and Centre Hospitalier Universitaire Vaudois, Lausanne, Switzerland (S.P.).

1. Antonia SJ, Villegas A, Daniel D, et al. Durvalumab after chemoradiotherapy in stage III non-small-cell lung cancer. N Engl J Med 2017;377:1919-29.

2. Bezjak A, Temin S, Franklin G, et al. Definitive and adjuvant radiotherapy in locally advanced non-small-cell lung cancer: 
American Society of Clinical Oncology clinical practice guideline endorsement of the American Society for Radiation Oncology evidence-based clinical practice guideline. J Clin Oncol 2015; 33:2100-5.

3. Bradley JD, Paulus R, Komaki R, et al. Standard-dose versus high-dose conformal radiotherapy with concurrent and consolidation carboplatin plus paclitaxel with or without cetuximab for patients with stage IIIA or IIIB non-small-cell lung cancer (RTOG 0617): a randomised, two-by-two factorial phase 3 study. Lancet Oncol 2015;16:187-99.

4. Butts C, Socinski MA, Mitchell PL, et al. Tecemotide (L-BLP25) versus placebo after chemoradiotherapy for stage III non-smallcell lung cancer (START): a randomised, double-blind, phase 3 trial. Lancet Oncol 2014;15:59-68.

5. Mauguen A, Pignon JP, Burdett S, et al. Surrogate endpoints for overall survival in chemotherapy and radiotherapy trials in operable and locally advanced lung cancer: a re-analysis of meta- analyses of individual patients' data. Lancet Oncol 2013;14:61926.

6. Borghaei H, Paz-Ares L, Horn L, et al. Nivolumab versus docetaxel in advanced nonsquamous non-small-cell lung cancer. N Engl J Med 2015;373:1627-39.

7. Albain KS, Swann RS, Rusch VW, et al. Radiotherapy plus chemotherapy with or without surgical resection for stage III non-small-cell lung cancer: a phase III randomised controlled trial. Lancet 2009;374:379-86.

8. Brahmer J, Reckamp KL, Baas P, et al. Nivolumab versus docetaxel in advanced squamous-cell non-small-cell lung cancer. N Engl J Med 2015;373:123-35.

9. Abbosh C, Birkbak NJ, Wilson GA, et al. Phylogenetic ctDNA analysis depicts early-stage lung cancer evolution. Nature 2017; 545:446-51.

DOI: 10.1056/NEJMe1711430

Copyright (๑) 2017 Massachusetts Medical Society.

\title{
Tolvaptan and Autosomal Dominant Polycystic Kidney Disease
}

\author{
Julie R. Ingelfinger, M.D.
}

Approximately half the patients with autosomal dominant polycystic kidney disease (ADPKD), a condition due to deficiency in polycystin 1 or 2 , have end-stage kidney disease by 60 years of age., ${ }^{1,2}$ Once progression begins, the mean decline in the estimated glomerular filtration rate (GFR) is approximately 4 to $6 \mathrm{ml}$ per minute per $1.73 \mathrm{~m}^{2}$ of body-surface area per year.

Therapy to prevent deterioration of GFR in patients with ADPKD has been elusive, despite mechanistic studies targeting pathogenesis. In addition, the burden of therapy in patients who have other symptoms from their ADPKD - hypertension, abdominal fullness and pain from cysts, hematuria, urinary tract infections, and nephrolithiasis, for example - is substantial. ${ }^{3}$

The value of various dietary changes (high water intake, low-salt diet, and soy diet) has been investigated, but these changes have limited efficacy. As more is learned about aberrant polycystin trafficking and signaling and other contributors to disease progression, targeted therapy is becoming feasible. ${ }^{4}$ Medications that have been evaluated include renoprotective agents, such as angiotensin-converting-enzyme inhibitors and angiotensin-receptor blockers; drugs that specifically interfere with pathways involved in the growth of kidney cysts, such as vasopressinreceptor antagonists, somatostatin, and interrupters of the mechanistic target of rapamycin
(mTOR) pathway; and cytokine blockers. To date, studies of medications that inhibit the effects of vasopressin have been particularly encouraging.

Laboratory studies have shown that relatively high levels of cyclic adenosine monophosphate (cAMP) facilitate cystogenesis in patients with ADPKD. ${ }^{5}$ Vasopressin $V_{2}$-receptor antagonists decrease cAMP within cells and slow the decline in kidney function in animal models. ${ }^{2}$ Early clinical studies were encouraging, and a multicenter, placebo-controlled, double-blind trial (the Tolvaptan Efficacy and Safety in Management of Autosomal Dominant Polycystic Kidney Disease and Its Outcomes [TEMPO] 3:4 trial) ${ }^{6}$ showed that in patients with an estimated GFR of more than 60 $\mathrm{ml}$ per minute per $1.73 \mathrm{~m}^{2}$, tolvaptan slowed the rate of kidney growth (the primary outcome); it also slowed the decline in the estimated GFR. However, more participants in the tolvaptan group than in the placebo group discontinued the medication owing to adverse events $(23 \%$ vs. $14 \%$; events were most often related to polyuria, polydipsia, nocturia, and urinary frequency). Furthermore, tolvaptan led to more instances of increased liver-enzyme levels, although abnormalities resolved after the discontinuation of the drug. After the publication of the results of the TEMPO 3:4 trial, the European Medicines Agency and the Health Products and Food Branch of Health Canada approved tolvaptan for the prevention of 